\title{
Balloon Deflation Strategy during Primary Percutaneous Coronary Intervention in Acute ST-Segment Elevation Myocardial Infarction: A Randomized Controlled Clinical Trial and Numerical Simulation-Based Analysis
}

\author{
Jun Gu, ${ }^{1}$ Yang Zhuo, ${ }^{1}$ Tian-jiao Liu, ${ }^{1}$ Jie Li, ${ }^{2}$ Zhao-fang Yin, ${ }^{1}$ Zuo-jun Xu, ${ }^{1}$ Li Fan, ${ }^{1}$ Qing He, \\ Kan Chen, ${ }^{1}$ Hua-su Zeng, ${ }^{1}$ Xiao-fei Wang, ${ }^{1}$ Yu-qi Fan, ${ }^{1}$ Jun-feng Zhang, ${ }^{1}$ Fu-you Liang $\mathbb{D}^{2,3}$ \\ and Chang-qian Wang ${ }^{1}{ }^{1}$ \\ ${ }^{1}$ Department of Cardiology, Shanghai Ninth People's Hospital, Shanghai Jiaotong University School of Medicine, Shanghai, China \\ ${ }^{2}$ School of Naval Architecture, Ocean and Civil Engineering, Shanghai Jiao Tong University, Shanghai, China \\ ${ }^{3}$ Institute for Personalized Medicine, Sechenov University, Moscow, Russia
}

Correspondence should be addressed to Fu-you Liang; fuyouliang@sjtu.edu.cn and Chang-qian Wang; shxkliuxu@126.com

Received 12 May 2020; Revised 24 August 2020; Accepted 27 August 2020; Published 7 September 2020

Academic Editor: Somasundaram Raghavan

Copyright (C) 2020 Jun Gu et al. This is an open access article distributed under the Creative Commons Attribution License, which permits unrestricted use, distribution, and reproduction in any medium, provided the original work is properly cited.

\begin{abstract}
Background. Primary percutaneous coronary intervention (PCI) is the best available reperfusion strategy in patients with acute ST-segment elevation myocardial infarction (STEMI). However, PCI is associated with a serious problem known as no-reflow phenomenon, resulting in poor clinical and functional outcomes. This study aimed to compare the influences of different balloon deflation velocity on coronary flow and cardiovascular events during primary PCI in STEM as well as transient hemodynamic changes in in vitro experiments. Method and Results. 211 STEMI patients were randomly assigned to either a rapid or a slow balloon deflation group during stent deployment. The primary end point was coronary flow at the end of PCI procedure, and secondary end points included myocardial infarct size. Transient hemodynamic changes were evaluated through an in vitro experimental apparatus and a computer model. In clinical practice, the level of corrected TIMI frame count (cTFC) in slow balloon deflation after primary PCI was significantly lower than that of rapid balloon deflation, which was associated with smaller infarct size. Numerical simulations revealed that the rapid deflation led to a sharp acceleration of flow in the balloon-vessel gap and a concomitant abnormal rise in wall shear stress (WSS). Conclusion. This randomized study demonstrated that the slow balloon deflation during stent implantation improved coronary flow and reduced infarct size in reperfused STEMI. The change of flow in the balloon-vessel gap and WSS resulted from different balloon deflation velocity might be partly accounted for this results.
\end{abstract}

\section{Introduction}

Although timely and successful reperfusion with primary percutaneous coronary intervention (PCI) is the most effective method for reducing infarct size and improving the outcome in patients with acute ST-segment elevation myocardial infarction (STEMI) $[1,2], \mathrm{PCI}$ is also associated with a serious problem known as no-reflow phenomenon, which significantly attenuates the beneficial effects of reperfusion therapy, resulting in poor clinical and functional outcomes. This phenomenon may develop in $5-50 \%$ of
STEMI patients during primary PCI [3-6]. No-reflow is thought to be caused by a variety of pathophysiological factors, such as distal embolization, ischemia-reperfusion injury, and the susceptibility of coronary microcirculation to injury [3-6].

Optimal stent deployment is an important component in determining outcomes with primary PCI for STEMI. Currently, rapid balloon deflation (from higher inflation pressure abruptly to negative pressure) during coronary stent deployment is universally performed. However, the rapid stent balloon deflation might lead to more significant siphonic effects and rapid changes in coronary 
hemodynamics, which was probably associated with distal embolization and microcirculation dysfunction [7]. And the embolization of plaque debris may be an important cause of the no-reflow or slow-flow phenomenon [8]. Until now, there is a paucity of data to evaluate the association of balloon deflation strategies during stent deployment with coronary flow and clinical outcomes in patients presenting with STEMI treated with primary PCI. The primary finding of this study is that the use of slow deflation strategy led to favorable coronary flow and infarct size compared with conventional rapid deflation for stent deployment. Additionally, an in vitro experimental apparatus combined with a computer model indicated that the change of flow velocity in the balloon-vessel gap and wall shear stress (WSS) resulted from different balloon deflation strategy might be partly accounted for this clinical benefit.

\section{Methods}

2.1. Clinical Study Design and Patients. This was a singlecenter, prospective, randomized, and controlled study. After the patients had given informed consent, they were randomly allocated to either the rapid or slow balloon deflation group through pre-established sealed envelopes in a $1: 1$ ratio after diagnostic angiography. Imaging investigators, statisticians, and also patients were blinded to the allocated group. Numbered sealed envelopes that contained the study group assignment were distributed to each catheterization laboratory and were opened after informed consent had been obtained. The authors designed the study, and the local institutional review board approved the trial protocol. Inclusion criteria were as follows: (1) patients $\geq 18$ and $\leq 80$ years of age; (2) STEMI patients presenting within $12 \mathrm{~h}$ after chest pain onset, with ST-segment elevation $\geq 0.1 \mathrm{mV}$ in two contiguous leads on 12-lead electrocardiogram or new left bundle branch block; (3) target lesion in a native coronary vessel with a reference diameter of 2.5 to $4.0 \mathrm{~mm}$; (4) visual residual diameter stenosis $\geq 70 \%$ before stent implantation; (5) voluntary participation and signed informed consent. Exclusion criteria were (1) cardiogenic shock; (2) acute occlusive lesion of left main, bypass grafting, and in-stent; (3) high risk of bleeding or allergy to aspirin, heparin, clopidogrel, ticagrelor, or rapamycin; (4) complicated with other serious diseases (malignant tumor, organ transplantation, or candidate); (5) severe liver and kidney dysfunction; (6) noncardiac comorbid conditions with a life expectancy $<1$ year or that may result in protocol noncompliance (per site investigator's medical judgment).

PCI was performed according to standard techniques. Before the index procedure, all patients received $300 \mathrm{mg}$ aspirin and $600 \mathrm{mg}$ clopidogrel or $180 \mathrm{mg}$ ticagrelor as loading doses. Unfractionated heparin was administered intravenously before PCI, and the active clotting time was maintained at $>250$ seconds throughout the procedure. Thrombus aspiration, predilation before stenting or postdilation after stenting, or use of glycoprotein IIb/IIIa inhibitors was left to the operators' discretion. In the rapid deflation group, the stent balloon was deflated to negative pressure abruptly during stent deployment. In the slow deflation group, the stent balloon was deflated to zero atmospheres and then negative pressure slowly (2 atmospheres per second). This balloon deflation strategy was also applied in stent postdilation, if necessary. After reperfusion, PCI was completed according to the physician's judgment with respect to patient status.

2.2. Corrected TIMI Frame Count (cTFC). Diagnostic coronary angiography and PCI procedure were performed by the insertion of a 6-French arterial sheath via the radial artery using the Seldinger method. Angiography CDs of the patients were reviewed by two interventional cardiologists who were blinded to all data other than the coronary angiograms. TIMI frame count (TFC) was determined with a digital system in the catheterization laboratory. TFC refers to the numbers of cine-frames required for contrast to reach a standardized distal coronary landmark in the culprit vessel [9]. cTFC means that the TFC of left anterior descending (LAD) must be corrected by dividing it into 1.7 because of the longer length of LAD. The cTFC count is considered as 100 frames for an occluded vessel [10]. Enrolled patients received automated contrast injection with the ACIST device (ACIST Medical Systems Inc., Eden Prairie, MN) for cTFC evaluation.

After the procedure, creatine kinase $(\mathrm{CK}) / \mathrm{CK}-\mathrm{MB}$ and troponin I (TNI) were measured before PCI and every 6 hours for 24 hours after the index procedure, thereafter, CK$\mathrm{MB}$ and TNI were measured once daily to document the peak value. Besides, the area under the curve (AUC) of the release of $\mathrm{CK}-\mathrm{MB}$ was approximated as a surrogate marker of infarct size. GraphPad Prism 5.02 was used to measure the AUC. All patients were recommended to receive optimal pharmacological therapy, including statins, $\beta$-blockers, or renin-angiotensin system blockade, following the current guidelines. Dual antiplatelet therapy (aspirin $100 \mathrm{mg} / \mathrm{d}$ plus clopidogrel $75 \mathrm{mg} / \mathrm{d}$ or ticagrelor $90 \mathrm{mg} / \mathrm{bid}$ ) was recommended for at least 12 months.

2.3. Study End Points. The primary end point was coronary flow (determined by cTFC) after the procedure. Secondary end points included myocardial infarct size and major adverse cardiac events (MACEs, a composite of death, myocardial infarction, revascularization, heart failure, and rehospitalization) at 30 days. No-reflow was defined as the culprit coronary artery flow less than TIMI flow grade 2 during or at the end of the PCI as revealed by coronary angiogram.

\subsection{Hemodynamic Characteristics upon Different Balloon} Deflation Strategy. On the basis of our previous hemodynamic study $[11,12]$, we innovatively established a hemodynamic in vitro study model of coronary balloon deflation [13]. An in vitro experimental apparatus was built, in which a high-speed camera was used to take snapshots of balloon deformation and flow field (dyed water) during balloon deflation. Subsequently, image processing techniques were employed to derive the parameters of balloon deformation 
and estimate the flow velocity downstream from the balloon (Figure 1). A computer model of the experimental apparatus was constructed, with the incorporation of the measured balloon deformation data, simulated the balloon deflation process under various perfusion pressure and fluid conditions. The basic reference velocity of balloon deformation (vb) was measured by in vitro balloon deformation experiment $\left(P_{\text {in }}=120 \mathrm{mmHg}\right.$ and $P_{\text {out }}=20 \mathrm{mmHg}$ ) [13-15]. In the numerical calculation, vb was reduced by $50 \%$ and increased by $100 \%$ respectively to simulate the different conditions of balloon deflation.

2.5. Statistical Analysis. To test the hypothesis that slow stent balloon deflation adjunctive to primary PCI is superior to conventional rapid balloon deflation for improving coronary reperfusion, we assumed that slow deflation would decrease the cTFC by 4 based on our previous work. On the basis of the estimated improvement in the primary end point, we selected a target sample size of at least 192 subjects, which would provide $80.0 \%$ power at the 0.05 significance level to detect anticipated differences and to offer some protection for coronary flow. Statistical analysis was performed using SPSS Statistical Software, version 22.0 (SPSS Inc., Chicago, IL, USA). Arithmetic means \pm standard deviations were calculated for quantitative variables, while qualitative variables were given as frequency and percentage (\%). For quantitative variable analysis, the $t$-test was used. A twosided chi-square test was used to compare qualitative variables. Differences in clinical endpoints between rapid and slow balloon deflation were tested with the $t$-test or chisquared test. All values were two-tailed, and a $P$ value $<0.05$ was considered statistically significant.

\section{Results}

3.1. Study Population. Between August 2015 and January 2018, 458 patients were considered to be eligible for the present study. Of these, 247 patients were not included for the following reasons: previous PCI or coronary artery bypass graft $(\mathrm{CABG})(n=25)$, beyond the age range $(n=68)$, with reference diameter beyond the range of 2.5 to $4.0 \mathrm{~mm}$ $(n=51)$, cardiogenic shock $(n=32)$, severe kidney dysfunction $(n=3)$, or involuntary participation $(n=87)$ (Figure 2). Data were thus presented for 211 patients (105 in the rapid balloon deflation group, and 106 in the slow balloon deflation group). There was no difference between the two groups with respect to the baseline population characteristics (Table 1). Duration of ischemia, coronary angiography features, and treatments administered either before and during PCI or at discharge were comparable between groups (Tables 2 and 3 ).

3.2. Coronary Flow and Infarct Size. For the primary endpoint, final CTFC after primary PCI in the slow balloon deflation group decreased significantly compared to the rapid balloon deflation group ( $24 \pm 7$ vs $27 \pm 9, P=0.015)$. In addition, the change of cTFC from baseline was much more prominent in the slow deflation $(65.9 \pm 16.5$ vs $61.0 \pm 18.2$,
$P=0.045)$. And considerable improvements of coronary flow (cTFC) were observed in the slow deflation compared with the rapid deflation immediately after stent deployment ( $25 \pm 8$ vs $28 \pm 9, P=0.035$ ). Although there was less "noreflow" in the slow deflation relative to the rapid deflation, no significant difference was found $(3 / 106$ vs $8 / 105, P=0.118)$. Finally, the peak for serum CK/CK-MB or TNI release was significantly lower in the slow deflation versus that in the rapid deflation (Table 4). And the AUC of CK-MB (0-72 h) in the slow deflation was significantly less than that in the rapid deflation (8302.2 \pm 3916.5 vs $9570.7 \pm 5122.3, P=0.022)$.

3.3. Left Ventricular Ejection Fraction and MACEs. Echocardiography was performed at 7 days after reperfusion. Regarding the left ventricular ejection fraction (LVEF), there was no obvious difference between the two groups (slow vs rapid: $51.9+8.4 \%$ vs $50.1+7.9 \%, P=0.089$ ). Compared to the rapid balloon deflation, the slow balloon deflation did not lead to lower rates of 30-day MACEs events (Table 5 and Figure 3).

\subsection{Hemodynamic Characteristics by Numerical Simulations.} Numerical simulations revealed that under the condition of $\mathrm{vb}$, about $0.18 \mathrm{~s}$ after the balloon deflation, the WSS downstream of the balloon reached $1.75 \mathrm{~Pa}$, close to the physiological value of blood flow velocity in the coronary artery [15]; however, the WSS (110-115 Pa) in the area of balloon reached over 60 times of their physiological values (Figure 4(a)). Moreover, rapid balloon deflation led to a sharp acceleration of flow in the balloon-vessel gap (Figures 4(c) and 4(d)) and a concomitant abnormal rise in WSS (Figure 4(b)).

\section{Discussion}

In this prospective, randomized, and controlled trial, the slow balloon deflation during stent deployment with primary PCI improved coronary flow and decreased infarct size to a greater extent than conventional rapid deflation strategy. However, the rate of 30-day MACEs was not significantly different between the two groups. The favorable effect of slow balloon deflation might be associated with its influence on hemodynamic characteristics.

STEMI remains one of the leading causes of death worldwide. The main goal in the treatment of STEMI is to recanalize the culprit artery occlusion at an early stage. Primary PCI has been shown to be the most effective reperfusion strategy in the treatment of STEMI. When noreflow occurs during primary PCI, it significantly attenuates the beneficial impact of reperfusion therapy, resulting in poor clinical and functional outcomes. Previous studies indicate that no-reflow is a multifactorial phenomenon, and its mechanisms include pre-existing microvascular dysfunction, distal micro-thrombo-embolization, ischemic injury, reperfusion injury, and individual susceptibility [3-6].

The restoration of coronary blood flow in STEMI reperfusion can paradoxically induce additional myocardial damage. The characteristics of clinically identified features of 

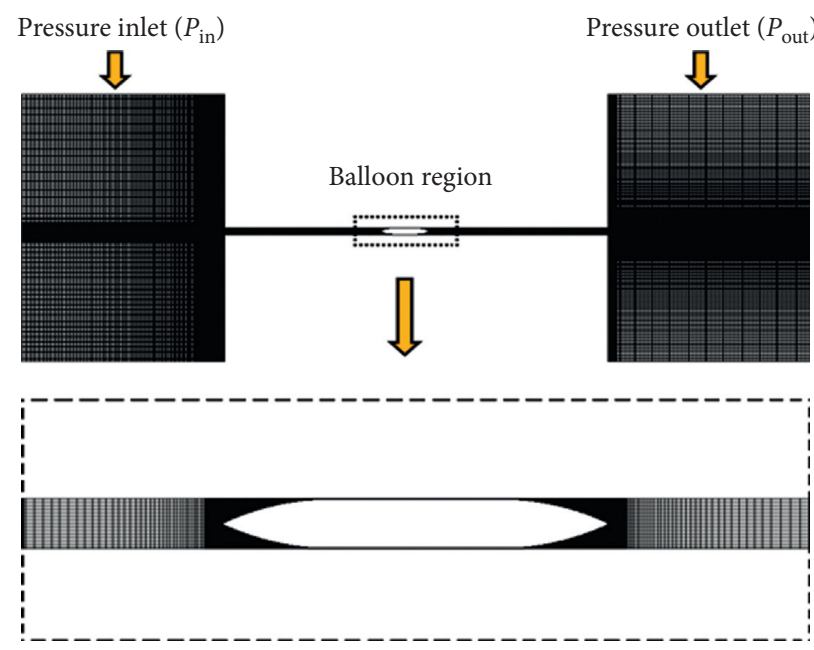

Figure 1: Computational mesh model.

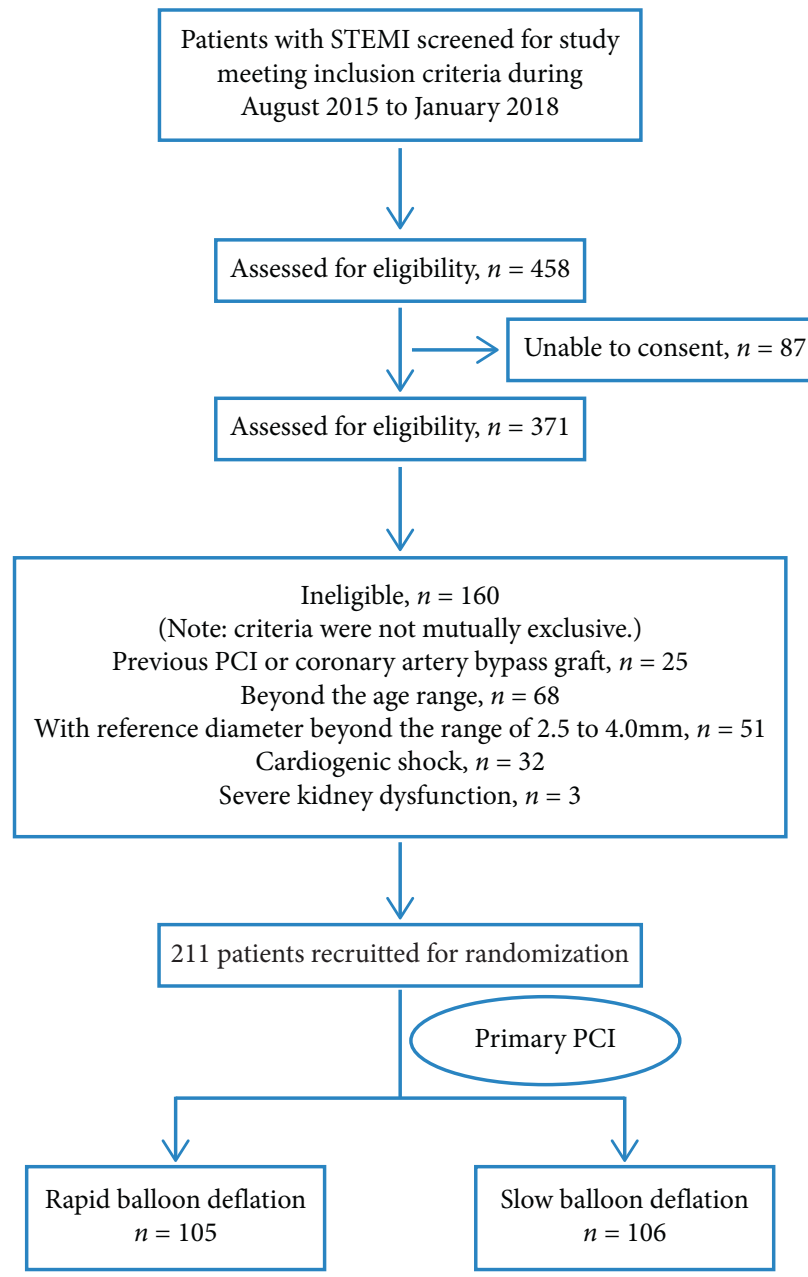

FIGURE 2: Flowchart of the clinical study protocol.

this reperfusion injury might be reversible and transient, such as arrhythmias or myocardial stunning, or irreversible, such as myocardial infarction or microvascular obstruction [16]. The myocardial damage during PCI might be associated with balloon deflation strategy during stent deployment. During stent implantation, the rapid deflation of the stent balloon might lead to increased coronary blood flow volatility and local shear stress, which was probably related with 
TABLE 1: Baseline characteristics.

\begin{tabular}{|c|c|c|c|}
\hline & Rapid deflation, $n=105$ & Slow deflation, $n=106$ & $P$ \\
\hline Age (years) & $62.9 \pm 13.7$ & $61.2 \pm 10.9$ & 0.322 \\
\hline Male (gender) & $84(80.0)$ & $92(86.8)$ & 0.185 \\
\hline $\operatorname{BMI}\left(\mathrm{kg} / \mathrm{m}^{2}\right)$ & $24.7 \pm 2.2$ & $24.4 \pm 2.4$ & 0.332 \\
\hline $\mathrm{SBP}(\mathrm{mmHg})$ & $125 \pm 25$ & $124 \pm 19$ & 0.740 \\
\hline $\operatorname{DBP}\left(\mathrm{kg} / \mathrm{m}^{2}\right)$ & $76 \pm 12$ & $75 \pm 11$ & 0.656 \\
\hline Oxygen saturation (\%) & $97.9 \pm 1.9$ & $98.3 \pm 1.7$ & 0.216 \\
\hline Hemoglobin $(\mathrm{g} / \mathrm{l})$ & $135.7 \pm 16.8$ & $137.5 \pm 16.1$ & 0.433 \\
\hline $\mathrm{BNP}(\mathrm{pg} / \mathrm{ml})$ & $190 \pm 229$ & $160 \pm 146$ & 0.267 \\
\hline Hypertension & $62(59.0)$ & $57(53.8)$ & 0.468 \\
\hline Hyperlipoidemia & $34(32.4)$ & $28(26.4)$ & 0.544 \\
\hline Diabetes & $20(19.0)$ & $21(19.8)$ & 0.889 \\
\hline Smoking & $55(52.4)$ & $59(55.7)$ & 0.631 \\
\hline $\operatorname{eGFR}\left(\mathrm{ml} / \mathrm{min} / 1.73 \mathrm{~m}^{2}\right)$ & $59.5+8.8$ & $60.4 .9+8.6$ & 0.455 \\
\hline
\end{tabular}

Data are presented as mean \pm SD or number (\%) of subjects. BMI: body mass index; SBP: systolic blood pressure; DBP: diastolic blood pressure; eGFR: estimated glomerular filtration rate; BNP: brain natriuretic peptide.

TABle 2: Angiographic characteristics.

\begin{tabular}{|c|c|c|c|}
\hline & Rapid deflation, $n=105$ & Slow deflation, $n=106$ & $P$ \\
\hline \multicolumn{4}{|l|}{ Infarct-related artery } \\
\hline LAD & $45(42.9)$ & $56(52.8)$ & 0.147 \\
\hline LCX & $22(21.0)$ & $14(13.2)$ & 0.135 \\
\hline RCA & $38(36.2)$ & $36(34.0)$ & 0.735 \\
\hline \multicolumn{4}{|l|}{ Coronary lesion } \\
\hline One-vessel & $65(61.9)$ & $62(58.2)$ & 0.612 \\
\hline Two-vessel & $31(29.5)$ & $35(33.0)$ & 0.584 \\
\hline Three-vessel & $9(8.5)$ & $9(8.5)$ & 0.983 \\
\hline \multicolumn{4}{|l|}{ Killip classification } \\
\hline I/II/III/IV & $88 / 16 / 1 / 0$ & $89 / 15 / 2 / 0$ & 0.833 \\
\hline Symptom to FMC (h) & $4.6 \pm 3.4$ & $4.1 \pm 3.2$ & 0.264 \\
\hline $\mathrm{D}$ to $\mathrm{B}(\mathrm{min})$ & $74 \pm 35$ & $77 \pm 34$ & 0.481 \\
\hline
\end{tabular}

Data are presented as mean \pm SD or number (\%) of subjects. LAD: left anterior descending; LCX: left circumflex; RCA: right coronary artery; FMC: first medical contact; D to B: door to balloon.

TABle 3: PCI characteristics and medications.

\begin{tabular}{|c|c|c|c|}
\hline & Rapid deflation, $n=105$ & Slow deflation, $n=106$ & $P$ \\
\hline Target lesion length (mm) & $24.4 \pm 9.3$ & $24.2 \pm 9.5$ & 0.876 \\
\hline Proximal reference $(\mathrm{mm})$ & $3.3 \pm 0.5$ & $3.4 \pm 0.4$ & 0.302 \\
\hline Distal reference $(\mathrm{mm})$ & $3.1 \pm 0.4$ & $3.2 \pm 0.4$ & 0.391 \\
\hline Number of stent implanted & $1.13 \pm 0.34$ & $1.13 \pm 0.34$ & 0.979 \\
\hline Inflation pressure during stent implantation (atm) & $16.2 \pm 2.1$ & $16.1 \pm 2.0$ & 0.570 \\
\hline Predilation & $92(87.6)$ & $90(84.9)$ & 0.567 \\
\hline Postdilation & $48(45.7)$ & $42(39.6)$ & 0.371 \\
\hline Thrombus aspiration & $21(20.0)$ & $24(22.6)$ & 0.640 \\
\hline Tirofiban & $77(73.3)$ & $73(68.9)$ & 0.474 \\
\hline aspirin + clopidogrel & $78(74.3)$ & $77(72.6)$ & 0.787 \\
\hline aspirin + ticagrelor & $27(25.7)$ & $29(27.4)$ & 0.787 \\
\hline ACEI/ARB & $92(87.6)$ & $93(87.7)$ & 0.979 \\
\hline Statins & $88(83.8)$ & $86(81.1)$ & 0.609 \\
\hline Beta-blockers & $90(85.7)$ & $92(86.8)$ & 0.820 \\
\hline Oral anticoagulation & $3(2.9)$ & $2(1.9)$ & 0.683 \\
\hline
\end{tabular}

Data are presented as mean \pm SD or number (\%) of subjects. ACEI/ARB: angiotensin-converting enzyme inhibitor/angiotensin II receptor blocker.

ischemia-reperfusion injury [16]. Our in vitro experiment and computer model indicated that WSS in the balloon area reached over 60 times of their physiological values during balloon deflation. More importantly, compared with the slow balloon deflation, the rapid deflation led to a sharp acceleration of flow in the balloon-vessel gap and a 
TABLE 4: Results of coronary flow.

\begin{tabular}{|c|c|c|c|}
\hline & Rapid deflation, $n=105$ & Slow deflation, $n=106$ & $P$ \\
\hline \multicolumn{4}{|l|}{ TIMI before PCI } \\
\hline $0 / 1 / 2 / 3$ & $79 / 10 / 8 / 8$ & $87 / 2 / 7 / 10$ & 0.111 \\
\hline CTFC before PCI (frames) & $88 \pm 23$ & $89 \pm 24$ & 0.867 \\
\hline CTFC before stenting (frames) & $43 \pm 18$ & $44 \pm 22$ & 0.654 \\
\hline CTFC post1 (frames) & $28 \pm 9$ & $25 \pm 8$ & 0.035 \\
\hline CTFC post2 (frames) & $27 \pm 9$ & $24 \pm 7$ & 0.015 \\
\hline $\mathrm{CK} \max (\mu / \mathrm{l})$ & $4098 \pm 3410$ & $2847 \pm 2204$ & 0.004 \\
\hline CK-MB $\max (\mu / 1)$ & $357 \pm 236$ & $272 \pm 212$ & 0.007 \\
\hline TNI max (ng/ml) & $65.3 \pm 30.7$ & $52.4 \pm 33.6$ & 0.004 \\
\hline No-reflow & $8(7.6)$ & $3(2.8)$ & 0.118 \\
\hline
\end{tabular}

Data are presented as mean \pm SD or number (\%) of subjects. TIMI: thrombolysis in myocardial infarction; CTFC: corrected TIMI frame count; PCI: percutaneous coronary intervention; CTFC post1: CTFC immediately after stent deployment: CTFC post2: CTFC at the end of PCI; CK: creatine kinase, TNI: troponin.

TABLE 5: MACEs at 30-day.

\begin{tabular}{|c|c|c|c|}
\hline & Rapid deflation, $n=105$ & Slow deflation, $n=106$ & $P$ \\
\hline Mortality & $3(2.9)$ & $2(1.9)$ & 0.683 \\
\hline Heart failure & $6(5.7)$ & $7(6.6)$ & 0.788 \\
\hline Myocardial infarction & $1(1.0)$ & 0 & 0.498 \\
\hline Target vessel/lesion revascularization & $1(1.0)$ & $1(0.9)$ & 1.000 \\
\hline Rehospitalization & $10(9.5)$ & $9(8.5)$ & 0.793 \\
\hline
\end{tabular}

Data are presented as number (\%) of subjects.

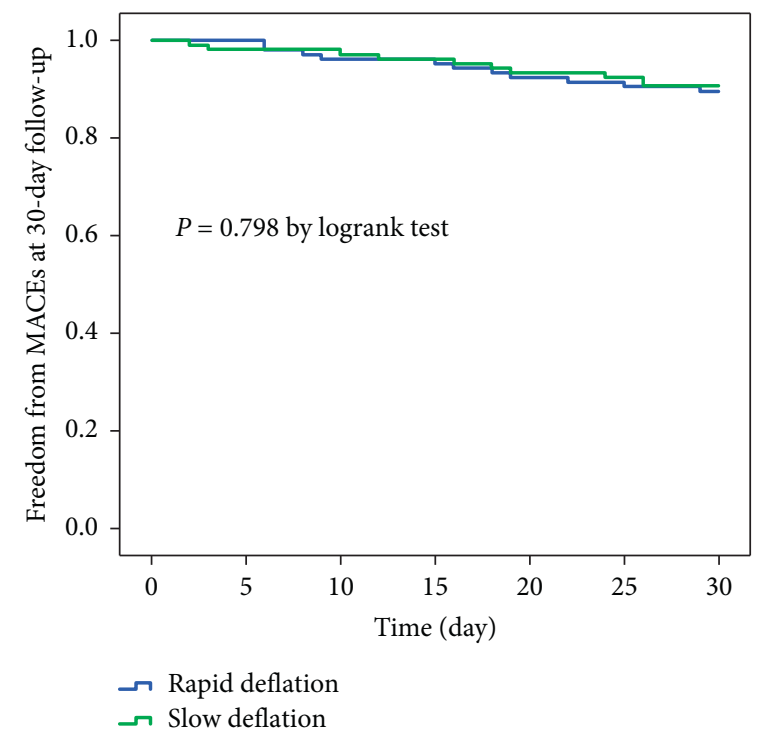

$\begin{array}{cccccccc}\begin{array}{c}\text { Rapid } \\ \text { deflation } \\ \begin{array}{c}\text { Slow } \\ \text { deflation }\end{array}\end{array} & 105 & 105 & 101 & 100 & 97 & 95 & 94 \\ & 103 & 102 & 99 & 98 & 96\end{array}$

FIGURE 3: Kaplan-Meier curves of freedom from the occurrence of major adverse cardiovascular events (MACEs) for different balloon deflation strategy during 30-day follow-up.

concomitant abnormal rise in WSS. In theory, the rapid balloon deflation might increase the risk of falling off of plaque fragments and microcirculation embolization in the downstream of coronary artery.
Previous studies have reported that high WSS is closely related to more vulnerable plaque [17]. High WSS assessed by intravascular ultrasound images is also associated with longitudinal development of high-risk plaque, including 


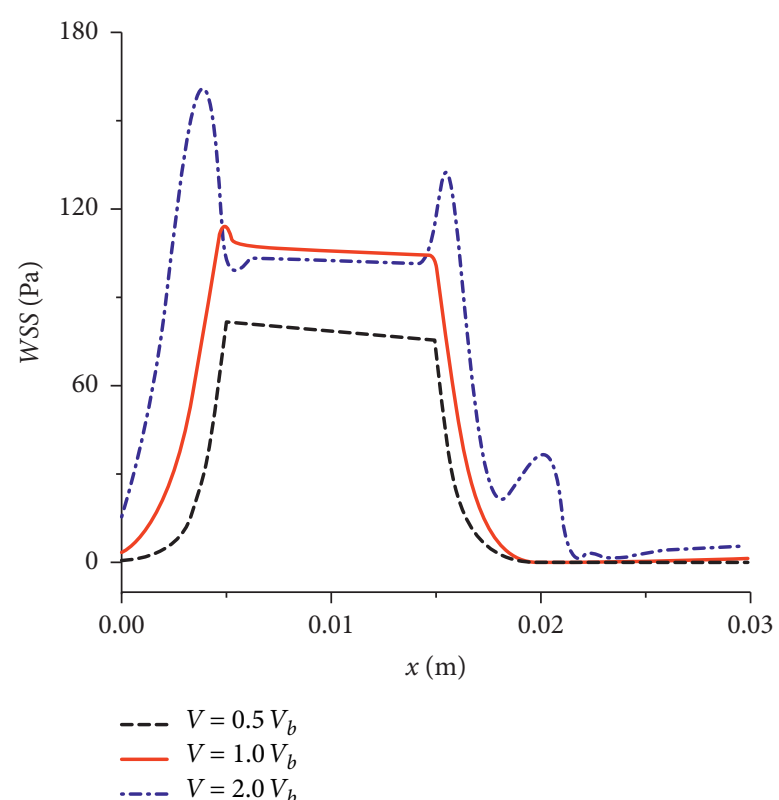

(a)

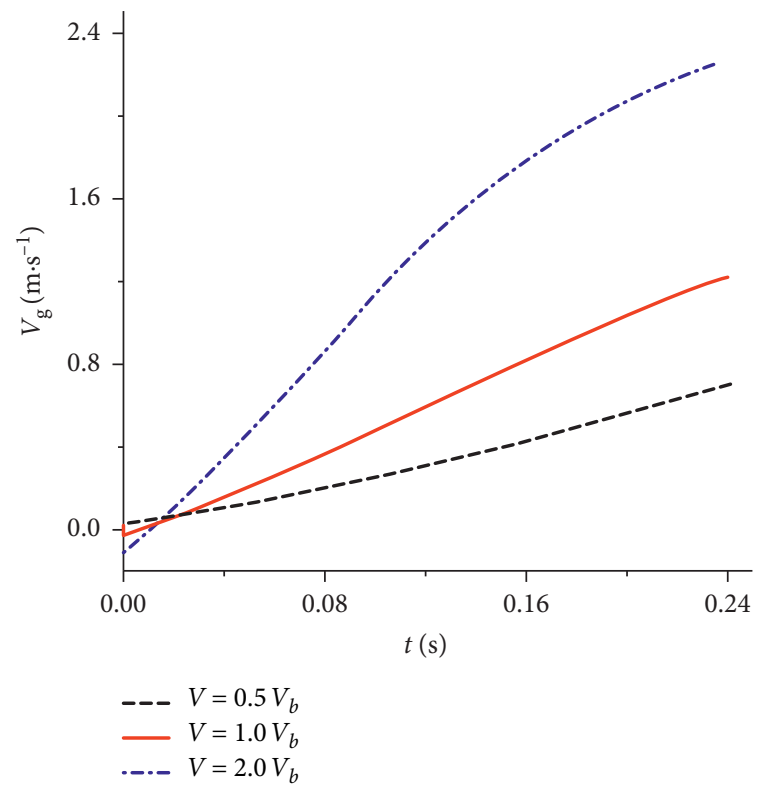

(c)

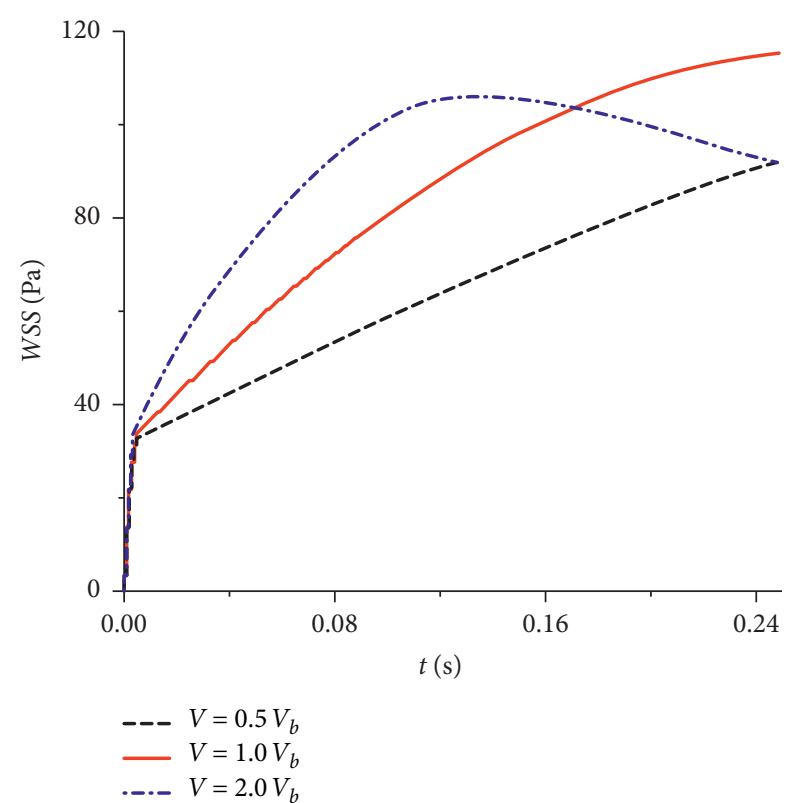

(b)

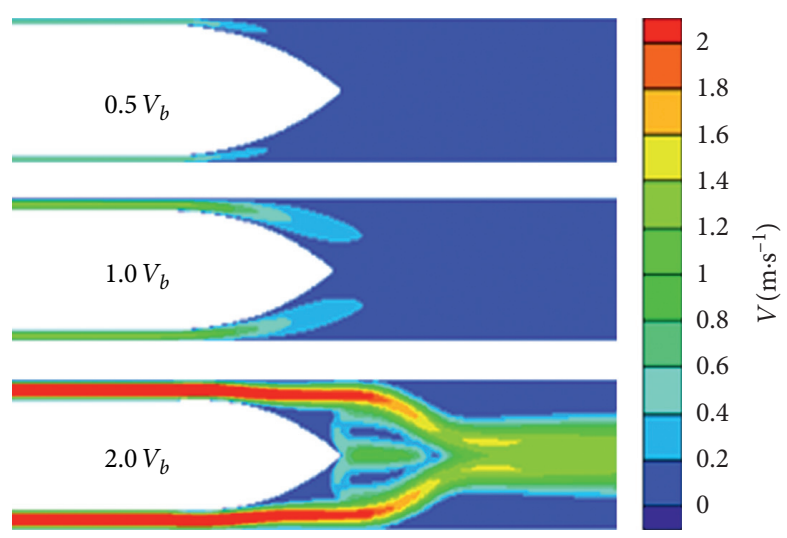

(d)

FIGURE 4: Comparisons of simulated flow velocities and vascular wall shear stress (WSS) under various balloon deflation velocities. (a) Simulated vascular WSS with the fluid and perfusion pressure along the balloon; (b) simulated vascular WSS in the balloon area under different velocity of balloon deflation; (c) simulated flow velocity in the balloon-vessel gap under different velocity of balloon deflation; (d) simulated flow velocity contour maps under different velocity of balloon deflation.

intraplaque necrotic core or expansive remodeling $[15,18,19]$. Indeed, higher WSS in the proximal segments of atherosclerotic lesions is predictive of myocardial infarction in patients with stable coronary artery disease (CAD) $[18,20]$. During primary PCI for STEMI, the rapid balloon deflation leads to higher WSS, which might be associated with adverse cardiovascular events. Moreover, fluctuations in coronary flow during reperfusion result in flow disturbances, in parallel, endothelial proinflammatory activation and vascular leakage occur $[21,22]$. One study indicated that, and to what extent, the changes in WSS resulting from the loss as well as the subsequent regaining of blood flow during shock and resuscitation accounted for endothelial activation [7]. The abrupt reflow-related enhancement of cytokineinduced endothelial proinflammatory activation supported the view that sudden regain of flow during resuscitation had an aggravating effect on endothelial activation, which might play a significant role in vascular dysfunction and consequent organ injury [7].

Furthermore, myocardial edema initiates during the ischemic stage but abruptly expands during the first minutes of reperfusion when the gradient between the hyperosmotic 
extravascular fluid and the normo-osmotic blood rapidly increases [1]. Through increasing the hydrostatic pressure within the interstitial space, this edema can lead to capillary compression and aggravation of cell damage [23]. Myocardial edema is both a consequence and a mechanism of reperfusion injury through a vicious cycle.

Optimal stent deployment is an important component in determining outcomes with PCI. Since the seminal work by Colombo et al., high pressure stent balloon inflation has been the standard method of stent deployment [24]. Use of the pressure optimization protocol during stent balloon inflation in a recent study was associated with better longterm outcomes, particularly for target vessel revascularization [25]. However, duration of stent deflation, and particularly the velocity of balloon deflation, has not received as much attention. In the current study, the slow deflation improves coronary flow and shrinks the size of myocardial infarction, which might be associated with decreased coronary blood flow fluctuation or WSS and might exert a favorable effect on endothelial function, myocardial edema, and microvascular obstruction.

Although recent meta-analyses suggest a clinical benefit of postconditioning in reducing infarct size by serial cardiac enzymes, many subsequent larger trials using advanced cardiac MRI imaging have found no acute benefit of this intervention $[3,26]$. Therefore, it is unclear whether these potential improvements in surrogate markers translate into beneficial clinical outcome $[3,26]$. The success of postconditioning has been associated with patient-related factors (age and gender), stenting technique (direct stenting versus predilatation), aspiration thrombectomy, and the postconditioning algorithm itself (duration of the ischemia/ reperfusion cycles, time between reperfusion, and ischemic conditioning). On the other hand, a previous study indicated that ischemic postconditioning did not improve LVEF within 7 days but did improve it significantly over 3 months $[27,28]$. Consistent with this result, our data showed that the slow balloon deflation strategy, in spite of its favorable effects on decreasing infarct size (determined by CK and TNI release), did not lead to a significant improvement for LVEF during the early period following primary PCI. And myocardial stunning might mask the beneficial effects of contractile function within days to weeks after acute myocardial infarction [28]. Therefore, more long-term follow-up data might be needed.

Actually, the concept of ischemic postconditioning is much different from the concept of slow deflation during stenting in the present study, as stenting and thus slow balloon deflation is applied at a time point after reperfusion has been established. Deferred stenting, another attempt to improve the primary PCI outcome after reperfusion restored, is still controversial for the prevention of no-flow/ slow-flow or MACEs [29-33]. Therefore, more effectively adjunctive therapy is needed to enhance the benefits of primary PCI.

4.1. Study Limitations. First, this study was limited by the relatively small population of the STEMI patient from a single center. Due to the absence of similar studies to date, further research is required to establish the effectiveness of slow balloon deflation during stent deployment, with a larger sample size. Second, the myocardial infarct size was evaluated by the peak of CK/CK-MB or TNI and AUC of CK$\mathrm{MB}$ in the present study; however, cardiac MRI had not used to examine the myocardial edema or infarct size. Third, more than $70 \%$ enrolled patients have dual antiplatelet therapy with clopidogrel, and this does not correspond to the treatment recommended by the most recent guidelines for STEMI patients. The reason is that ticagrelor had not yet entered the list of China's medical insurance and was selffunded during the study period. Fourth, we speculated that the rapid balloon deflation might increase the risk of falling off of plaque fragments and microcirculation embolization in the downstream of coronary artery. However, the downstream filter wire was not utilized in the present study. Fifth, the abrupt opening of the complete occluded infarction-related artery (IRA) will have a greater hemodynamic effect on the downstream vessels. We speculated that the slow balloon deflation strategy during primary PCI might have favorable effects in STEMI patients with TIMI grade 0 in IRA, and patients with partial reperfusion might weaken the benefit of slow balloon deflation. Among the patients we enrolled, in spite of not all enrolled patients with IRA TIMI grade 0, a majority of IRA (166/211) are completely occluded, in which 79/105 in the rapid balloon deflation vs $87 / 106$ in the slow deflation $(P=0.967)$. As for the predilation during primary PCI, more than $80 \%$ lesions of IRA received predilation before stent implantation. And distal flow/pressure and WSS might be changed during the predilation procedure. However, from our clinical experience, compared with stent implantation or postdilation, balloon predilation causes less incidence of slow-flow or noreflow. And there was no significant difference with regard to the proportion of predilation procedure and cTFC value just before stenting between the two groups. Sixth, in general, there was no statistical difference in baseline data between the two groups (Table 1). However, in the BNP level, the proportions of hypertension and hyperlipidemia in the rapid balloon deflation group were slightly higher than that in the slow group, which might have a slight influence on the results. Lastly, the slow deflation did not reduce 30-day cardiovascular events, which might be associated with fewer patients enrolled and shorter duration of follow-up. Long-term follow-up is needed to assess the influence of slow balloon deflation on MACEs.

\section{Conclusions}

This randomized controlled trial demonstrated that the slow balloon deflation during stent implantation in primary PCI improved the coronary flow and decreased infarct size in patients with STEMI. This beneficial impact was likely related to decreased coronary flow volatility or shear stress.

\section{Data Availability}

The data that support the findings of this study are available on request from the corresponding author. 


\section{Conflicts of Interest}

The authors declare that they have no conflicts of interest.

\section{Authors' Contributions}

Jun Gu, Yang Zhuo, and Tian-jiao Liu contributed equally to this work.

\section{Acknowledgments}

This study was supported by Clinical Research Program of 9th People's Hospital affiliated to Shanghai Jiaotong University School of Medicine (JYLJ017 and JYLJ201803), research projects from Shanghai Science and Technology Commission (18411950500), and The Project of Construction and Application of Biobank for Coronary Heart Disease of Shanghai Ninth People's Hospital (YBKA201910).

\section{References}

[1] B. Ibanez, S. James, S. Agewall et al., "2017 ESC Guidelines for the management of acute myocardial infarction in patients presenting with ST-segment elevation: the Task Force for the management of acute myocardial infarction in patients presenting with ST-segment elevation of the European Society of Cardiology (ESC)," European Heart Journal, vol. 39, no. 2, pp. 119-177, 2018.

[2] J. Y. Hahn, Y. B. Song, E. K. Kim et al., "Ischemic postconditioning during primary percutaneous coronary intervention: the effects of postconditioning on myocardial reperfusion in patients with ST-segment elevation myocardial infarction (POST) randomized trial," Circulation, vol. 128, no. 17, pp. 1889-1896, 2013.

[3] L. S. F. Konijnenberg, P. Damman, D. J. Duncker et al., "Pathophysiology and diagnosis of coronary microvascular dysfunction in ST-elevation myocardial infarction," Cardiovascular Research, vol. 116, no. 4, pp. 787-805, 2020.

[4] A. Bayramoğlu, H. Taşolar, A. Kaya et al., "Prediction of noreflow and major adverse cardiovascular events with a new scoring system in STEMI patients," Journal of Interventional Cardiology, vol. 31, no. 2, pp. 144-149, 2018.

[5] C. Bouleti, N. Mewton, and S. Germain, "The no-reflow phenomenon: state of the art," Archives of Cardiovascular Diseases, vol. 108, no. 12, pp. 661-674, 2015.

[6] M. Dong, N. Mu, F. Guo et al., "The beneficial effects of postconditioning on no-reflow phenomenon after percutaneous coronary intervention in patients with ST-elevation acute myocardial infarction," Journal of Thrombosis and Thrombolysis, vol. 38, no. 2, pp. 208-214, 2014.

[7] R. Li, J. G. Zijlstra, J. A. A. M. Kamps, M. van Meurs, and G. Molema, "Abrupt reflow enhances cytokine-induced proinflammatory activation of endothelial cells during simulated shock and resuscitation," Shock, vol. 42, no. 4, pp. 356-364, 2014.

[8] M. Nishio, Y. Ueda, K. Matsuo et al., "Association of target lesion characteristics evaluated by coronary computed tomography angiography and plaque debris distal embolization during percutaneous coronary intervention," Circulation Journal, vol. 78, no. 9, pp. 2203-2208, 2014.

[9] K. Hibi, K. Kozuma, S. Sonoda et al., "A randomized study of distal filter protection versus conventional treatment during percutaneous coronary intervention in patients with attenuated plaque identified by intravascular ultrasound," JACC: Cardiovascular Interventions, vol. 11, no. 16, pp. 1545-1555, 2018.

[10] C. M. Gibson, S. A. Murphy, M. J. Rizzo et al., "Relationship between TIMI frame count and clinical outcomes after thrombolytic administration," Circulation, vol. 99, no. 15, pp. 1945-1950, 1999.

[11] X. Ge, Z. Yin, Y. Fan, Y. Vassilevski, and F. Liang, "A multiscale model of the coronary circulation applied to investigate transmural myocardial flow," International Journal for $\mathrm{Nu}$ merical Methods in Biomedical Engineering, vol. 34, no. 10, Article ID e3123, 2018.

[12] F. Liang, H. Senzaki, Z. Yin et al., "Transient hemodynamic changes upon changing a BCPA into a TCPC in staged Fontan operation: a computational model study," Scientific World Journal, vol. 2013, Article ID 486815, 10 pages, 2013.

[13] J. Li, Z. F. Yin, and F. Y. Liang, "In vitro experiment and numerical simulation based-study on the transient hemodynamic characteristics upon balloon deflation in coronary interventional operation," Journal of Medical Biomechanic, vol. 34, pp. 473-480, 2019.

[14] S. Nanto, T. Masuyama, Y. Takano, M. Hori, and S. Nagata, "Determination of coronary zero flow pressure by analysis of the baseline pressure-flow relationship in humans," Japanese Circulation Journal, vol. 65, no. 9, pp. 793-796, 2001.

[15] H. Samady, P. Eshtehardi, M. C. McDaniel et al., "Coronary artery wall shear stress is associated with progression and transformation of atherosclerotic plaque and arterial remodeling in patients with coronary artery disease," Circulation, vol. 124, no. 7, pp. 779-788, 2011.

[16] A. Prasad, G. W. Stone, D. R. Holmes, and B. Gersh, "Reperfusion injury, microvascular dysfunction, and cardioprotection," Circulation, vol. 120, no. 21, pp. 2105-2112, 2009.

[17] D. Shishikura, S. L. Sidharta, S. Honda et al., "The relationship between segmental wall shear stress and lipid core plaque derived from near-infrared spectroscopy," Atherosclerosis, vol. 275 , pp. $68-73,2018$.

[18] A. Kumar, E. W. Thompson, A. Lefieux et al., "High coronary shear stress in patients with coronary artery disease predicts myocardial infarction," Journal of the American College of Cardiology, vol. 72, no. 16, pp. 1926-1935, 2018.

[19] H. Hetterich, A. Jaber, M. Gehring et al., "Coronary computed tomography angiography based assessment of endothelial shear stress and its association with atherosclerotic plaque distribution in-vivo," PLoS One, vol. 101, Article ID e0115408, 2015.

[20] D. Han, A. Starikov, B. Ó Hartaigh et al., "Relationship between endothelial wall shear stress and high-risk atherosclerotic plaque characteristics for identification of coronary lesions that cause ischemia: a direct comparison with fractional flow reserve," Journal of the American Heart Association, vol. 5, Article ID e004186, 2016.

[21] M. van Meurs, F. M. Wulfert, A. J. Knol et al., "Early organspecific endothelial activation during hemorrhagic shock and resuscitation," Shock, vol. 29, pp. 291-299, 2008.

[22] E. W. Childs, B. Tharakan, N. Byrge, J. H. Tinsley, F. A. Hunter, and W. R. Smythe, "Angiopoietin-1 inhibits intrinsic apoptotic signaling and vascular hyperpermeability following hemorrhagic shock," American Journal of Physiology-Heart and Circulatory Physiology, vol. 294, no. 5, pp. H2285-H2295, 2008. 
[23] D. Garcia-Dorado and J. Oliveras, "Myocardial oedema: a preventable cause of reperfusion injury?" Cardiovascular Research, vol. 27, no. 9, pp. 1555-1563, 1993.

[24] A. Colombo, P. Hall, S. Nakamura et al., "Intracoronary stenting without anticoagulation accomplished with intravascular ultrasound guidance," Circulation, vol. 91, no. 6, pp. 1676-1688, 1995.

[25] S. Vallurupalli, S. Kasula, S. Kumar Agarwal et al., "A novel stent inflation protocol improves long-term outcomes compared with rapid inflation/deflation deployment method," Catheterization and Cardiovascular Interventions, vol. 90, no. 2, pp. 233-240, 2017.

[26] Z. Xing, L. Tang, J. Huang et al., "Effects of ischaemic postconditioning on outcomes of patients with ST-segment elevation myocardial infarction who underwent primary percutaneous coronary intervention: a meta-analysis," BMJ Open, vol. 9, Article ID e022509, 2019.

[27] Y. Wei, L. Ruan, G. Zhou et al., "Local ischemic postconditioning during primary percutaneous coronary intervention: a meta-analysis," Cardiology, vol. 123, no. 4, pp. 225-233, 2012.

[28] H. Thibault, C. Piot, P. Staat et al., "Long-term benefit of postconditioning," Circulation, vol. 117, no. 8, pp. 1037-1044, 2008.

[29] D. Carrick, K. G. Oldroyd, M. McEntegart et al., "A randomized trial of deferred stenting versus immediate stenting to prevent no- or slow-reflow in acute ST-segment elevation myocardial infarction (DEFER-STEMI)," Journal of the American College of Cardiology, vol. 63, no. 20, pp. 2088-2098, 2014.

[30] H. Kelbæk, D. E. Høfsten, L. Køber et al., “Deferred versus conventional stent implantation in patients with ST-segment elevation myocardial infarction (DANAMI 3-DEFER): an open-label, randomised controlled trial," The Lancet, vol. 387, pp. 2199-2206, 2016.

[31] J. Lønborg, T. Engstrøm, K. A. Ahtarovski et al., "Myocardial damage in patients with deferred stenting after STEMI: a DANAMI-3-DEFER substudy," Journal of the American College of Cardiology, vol. 69, pp. 2794-2804, 2017.

[32] L. Belle, P. Motreff, L. Mangin et al., "Comparison of immediate with delayed stenting using the minimalist immediate mechanical intervention approach in acute ST-segment-elevation myocardial infarction: the MIMI Study," Circulation: Cardiovascular Interventions, vol. 9, Article ID e003388, 2016.

[33] J. S. Kim, H. J. Lee, C. Woong Yu et al., "Innovation study (impact of immediate stent implantation versus deferred stent implantation on infarct size and microvascular perfusion in patients with ST-segment-elevation myocardial infarction)," Circulation: Cardiovascular Interventions, vol. 9, Article ID e004101, 2016. 